ESAIM: COCV 19 (2013) 828-843

DOI: $10.1051 / \mathrm{cocv} / 2012035$
ESAIM: Control, Optimisation and Calculus of Variations

www.esaim-cocv.org

\title{
PARTIALLY OBSERVED OPTIMAL CONTROLS OF FORWARD-BACKWARD DOUBLY STOCHASTIC SYSTEMS *
}

\author{
YufEng SHI ${ }^{1}$ AND QINGFENG ZHU ${ }^{2,1}$
}

\begin{abstract}
The partially observed optimal control problem is considered for forward-backward doubly stochastic systems with controls entering into the diffusion and the observation. The maximum principle is proven for the partially observable optimal control problems. A probabilistic approach is used, and the adjoint processes are characterized as solutions of related forward-backward doubly stochastic differential equations in finite-dimensional spaces. Then, our theoretical result is applied to study a partially-observed linear-quadratic optimal control problem for a fully coupled forward-backward doubly stochastic system.
\end{abstract}

Mathematics Subject Classification. 93E20, 60H10.

Received January 5, 2012. Revised August 22, 2012.

Published online June 3, 2013.

\section{INTRODUCTION}

It is well-known that the maximum principle, the necessary condition of the optimal controls, which is a milestone-like result in the optimal control theory, was established for the deterministic control systems by Pontryakin's group [18] in the 1950's and 1960's. Since then, a lot of work has been done on the forward stochastic control systems such as Bismut [6], Kushner [11], Peng [16] and Meng [14], etc. Maximum principles of forward-backward stochastic systems have been studied extensively in the literature. We refer to $[21,25,27]$ and references therein. They only established some necessary maximum principles in the cases the control domains are convex or the forward diffusion coefficients can not contain control variables.

In order to provide a probabilistic interpretation for the solutions of a class of semilinear stochastic partial differential equations (SPDEs in short), Pardoux and Peng [15] introduced the following backward doubly

\footnotetext{
Keywords and phrases. Forward-backward doubly stochastic system, partially observed optimal control, maximum principle, adjoint equation.

* This work was supported by National Natural Science Foundation of China (10771122, 11071145 and 11231005), Natural Science Foundation of Shandong Province of China (Y2006A08), Foundation for Innovative Research Groups of National Natural Science Foundation of China (10921101), National Basic Research Program of China (973 Program, 2007CB814900), Independent Innovation Foundation of Shandong University (2010JQ010), the 111 Project (B12023).

1 School of Mathematics, Shandong University, 250100 Jinan, P.R. China. yfshi@sdu.edu.cn

2 School of Mathematics and Quantitative Economics, Shandong University of Finance and Economics, 250014 Jinan, P.R. China.
} 
stochastic differential equations (BDSDEs in short):

$$
\begin{aligned}
Y(t)= & \xi+\int_{t}^{T} f(s, Y(s), Z(s)) \mathrm{d} s+\int_{t}^{T} g(s, Y(s), Z(s)) \overleftarrow{\mathrm{d}} B(s) \\
& -\int_{t}^{T} Z(s) \overrightarrow{\mathrm{d}} W(s), 0 \leq t \leq T
\end{aligned}
$$

Note that the integral with respect to $\{B(t)\}$ is a "backward Itô integral" and the integral with respect to $\{W(t)\}$ is a standard forward Itô integral. These two types of integrals are particular cases of the Itô-Skorohod integral (for details see [15]). Due to their important significance to SPDEs, the researches for BDSDEs have been in the ascendant (cf. Bally and Matoussi [3], Shi et al. [20], Zhang and Zhao [30,31], Ren et al. [19], Hu and Ren [9] and their references).

Peng and Shi [17] introduced a type of time-symmetric forward-backward stochastic differential equations, i.e. so-called fully coupled forward-backward doubly stochastic differential equations (FBDSDEs in short):

$$
\left\{\begin{aligned}
y(t)= & x+\int_{0}^{t} f(s, y(s), Y(s), z(s), Z(s)) \mathrm{d} s-\int_{0}^{t} z(s) \overleftarrow{\mathrm{d}} B(s) \\
& +\int_{0}^{t} g(s, y(s), Y(s), z(s), Z(s)) \overrightarrow{\mathrm{d}} W(s) \\
Y(t)= & \Phi(y(T))+\int_{t}^{T} F(s, y(s), Y(s), z(s), Z(s)) \mathrm{d} s+\int_{t}^{T} Z(s) \overrightarrow{\mathrm{d}} W(s) \\
& +\int_{t}^{T} G(s, y(s), Y(s), z(s), Z(s)) \overleftarrow{\mathrm{d}} B(s)
\end{aligned}\right.
$$

In FBDSDE (1.2), the forward equation is "forward" with respect to a standard stochastic integral $\overrightarrow{\mathrm{d}} W(t)$, as well as "backward" with respect to a backward stochastic integral $\overleftarrow{\mathrm{d}} B(t)$; the coupled "backward equation" is "forward" under the backward stochastic integral $\overleftarrow{\mathrm{d}} B(t)$ and "backward" under the forward one. In other words, both the forward equation and the backward one are types of BDSDE (1.1) with different directions of stochastic integrals. So (1.2) provides a very general framework of fully coupled forward-backward stochastic systems. Peng and Shi [17] proved the existence and uniqueness of solutions to FBDSDE (1.2) with arbitrarily fixed time duration under some monotone assumptions. FBDSDE (1.2) can provide a probabilistic interpretation for the solutions of a class of quasilinear SPDEs.

BDSDE and FBDSDEs can provide more extensive frameworks for stochastic Hamiltonian systems arising in stochastic optimal control problems. Recently, Han et al. [7] investigated the necessary condition of optimal controls and obtained a stochastic maximum principle for backward doubly stochastic optimal control systems. Almost simultaneously Bahlali and Gherbal [2] independently proved necessary and sufficient optimality conditions for backward doubly stochastic control systems. Complete information maximum principles of forward-backward doubly stochastic control systems of (1.2) have been studied in [28,29]. They established maximum principles in the case the control domain is convex or the diffusion coefficients can not contain a control variable.

However, in the control problems above, the information of the control problems is assumed to be completely observed. This is not reasonable in reality. Generally speaking, the controllers can only get partial information at most cases. This motivates us to study the partially observed optimal doubly stochastic control problems. The subject of stochastic maximum principles for partially-observed forward stochastic optimal control problems has been discussed by many authors, such as Bensoussan [5], Haussmann [8], Baras et al. [4], Li and Tang [12], Tang [23], etc. Li and Tang [12] obtained the maximum principle for the partially-observed forward stochastic optimal control problem in which the control variable is allowed to enter the diffusion and the observation coefficients. Moreover, the control domain is not necessarily convex. Tang [23] extended the result in [12] to the case with correlated noises between the system and the observation. A general maximum principle was proven for 
the partially-observed optimal control and the relations among the adjoint processes were established. Adjoint vector fields were introduced as the solutions to some backward stochastic partial differential equations (BSPDEs in short) and their relations were established. Under suitable conditions, the adjoint processes were characterized in terms of the adjoint vector fields, their differentials and Hessians, along the optimal state process. The optimal control problems for partially-observed forward-backward stochastic systems have been studied extensively in Shi and $\mathrm{Wu}[22]$, Wang and $\mathrm{Wu}[24]$ and $\mathrm{Wu}[26]$. They established maximum principles in the case the control domain is convex or the forward diffusion coefficients can not contain a control variable.

In this paper, we study the optimal control problems of partially-observed forward-backward doubly stochastic systems whose control domains are convex, but with the control variables capable of entering into the diffusion terms. We derive the maximum principle under some suitable conditions, which can be viewed as the counterpart of the results in Zhang and Shi [29] with complete observation. The main difficulty which we encounter is the partial observation. We use a pure probabilistic approach of Li and Tang [12]. Firstly, by Girsanov's theorem we reformulate our original partially-observed optimal control problem (2.1)-(2.5) to (2.1)-(2.3), (2.5) and (2.6), which is quite similar to a completely observed one. The only difference lies in the admissible control sets. One advantage of this approach is that it need neither the Zaka's equation as adopted in Haussmann [8] nor the theory of stochastic flows as adopted in Baras et al. [4]. So we get around the complicated stochastic calculus in infinite-dimensional spaces. Secondly, we obtain some estimations of the variational observed process and the difference between the perturbed observed process and the sum of the optimal and variational observed processes. Then we give the variational inequality. Finally, by the classical duality technique we prove the maximum principle.

To illustrate our theoretical results, we give an example for a partially-observed linear-quadratic (LQ in short) fully-coupled forward-backward doubly stochastic optimal control problem. Moreover, we can prove that the observable control variable is really optimal.

We emphasize that our problems should be distinguished from the optimal control problems with partial information, where a subfiltration is given to represent the information available to the controller instead of an observation process. For problems with partial information, there is a rich literature ( $c f$. Baghery and Øksendal [1], Huang et al. [10], Meng [13] and the references therein).

The rest of this paper is organized as follows. In Section 2, we state our partially observed optimal control problems and the main assumptions. In Section 3, we obtain the partially observed doubly stochastic maximum principle. Finally in Section 4, we discuss forward-backward doubly stochastic LQ optimal control problems to show the applications of the theoretical results.

\section{PReliminaries AND PROBlem FORMUlation}

Let $(\Omega, \mathcal{F}, P)$ be a completed probability space, $\{W(t)\}_{t \geq 0},\{B(t)\}_{t \geq 0}$ and $\{X(t)\}_{t \geq 0}$ be three mutually independent standard Brownian motions, with values respectively in $\mathbb{R}^{d}, \mathbb{R}^{l}$ and $\mathbb{R}^{r}$, defined on $(\Omega, \mathcal{F}, P)$. Let $\mathcal{N}$ denote the class of $P$-null sets of $\mathcal{F}$. For each $t \in[0, T]$, we define

$$
\begin{aligned}
& \mathcal{F}_{t}^{W} \doteq \sigma\{W(r) ; 0 \leq r \leq t\} \vee \mathcal{N}, \\
& \mathcal{F}_{t}^{X} \doteq \sigma\{X(r) ; 0 \leq r \leq t\} \vee \mathcal{N}, \\
& \mathcal{F}_{t, T}^{B} \doteq \sigma\{B(r)-B(t) ; t \leq r \leq T\} \vee \mathcal{N},
\end{aligned}
$$

and

$$
\mathcal{F}_{t} \doteq \mathcal{F}_{t}^{W} \vee \mathcal{F}_{t, T}^{B}, \forall t \in[0, T] .
$$

Note that $\left\{\mathcal{F}_{t}^{W} ; t \in[0, T]\right\}$ and $\left\{\mathcal{F}_{t}^{X} ; t \in[0, T]\right\}$ are increasing filtrations and $\left\{\mathcal{F}_{t, T}^{B} ; t \in[0, T]\right\}$ is an decreasing filtration, and the collection $\left\{\mathcal{F}_{t} ; t \in[0, T]\right\}$ is neither increasing nor decreasing. $\mathbb{E}$ denotes the expectation 
on $(\Omega, \mathcal{F}, P)$. We introduce the following notations:

$$
\begin{aligned}
M^{2}\left(0, T ; \mathbb{R}^{n}\right)= & \left\{v(t), 0 \leq t \leq T: v(t) \text { is an } \mathbb{R}^{n} \text {-valued, } \mathcal{F}_{t^{-} \text {-measurable process }}\right. \\
& \text { such that } \left.\mathbb{E} \int_{0}^{T}|v(t)|^{2} \mathrm{~d} t<\infty\right\}, \\
M_{\mathcal{F} X}^{2}\left(0, T ; \mathbb{R}^{n}\right)= & \left\{v(t), 0 \leq t \leq T: v(t) \text { is an } \mathbb{R}^{n} \text {-valued, } \mathcal{F}_{t}^{X}\right. \text {-measurable process } \\
& \text { such that } \left.\mathbb{E} \int_{0}^{T}|v(t)|^{2} \mathrm{~d} t<\infty\right\}, \\
L^{2}\left(\Omega, \mathcal{F}_{T}, P ; \mathbb{R}^{n}\right)= & \left\{\xi: \xi \text { is an } \mathbb{R}^{n} \text {-valued, } \mathcal{F}_{T}\right. \text {-measurable random variable } \\
& \text { such that } \left.\mathbb{E}|\xi|^{2}<\infty\right\}, \\
L^{2}\left(\Omega, \mathcal{F}_{T}^{X}, P ; \mathbb{R}^{n}\right)= & \left\{\xi: \xi \text { is an } \mathbb{R}^{n} \text {-valued, } \mathcal{F}_{T}^{X}\right. \text {-measurable random variable } \\
& \text { such that } \left.\mathbb{E}|\xi|^{2}<\infty\right\} .
\end{aligned}
$$

For a convex subset $U \subset \mathbb{R}^{k}$, let

$$
\mathcal{U}_{a d}[0, T]=\left\{v(t):[0, T] \times \Omega \rightarrow U \mid v(t) \text { is } \mathcal{F}_{t}^{X} \text {-measurable, } \mathbb{E} \int_{0}^{T}|v(t)|^{2} \mathrm{~d} t<\infty\right\} .
$$

For any given admissible control $v \in \mathcal{U}_{a d}$, we consider the following forward-backward doubly stochastic control system:

$$
\left\{\begin{aligned}
\mathrm{d} y(t)= & f(t, y(t), Y(t), z(t), Z(t), v(t)) \mathrm{d} t-z(t) \overleftarrow{\mathrm{d}} B(t) \\
& +g(t, y(t), Y(t), z(t), Z(t), v(t)) \overrightarrow{\mathrm{d}} W(t) \\
\mathrm{d} Y(t)= & -F(t, y(t), Y(t), z(t), Z(t), v(t)) \mathrm{d} t+Z(t) \overrightarrow{\mathrm{d}} W(t) \\
& -G(t, y(t), Y(t), z(t), Z(t), v(t)) \overleftarrow{\mathrm{d}} B(t) \\
y(0)= & x, \quad Y(T)=\Phi(y(T))
\end{aligned}\right.
$$

where $(y(\cdot), Y(\cdot), z(\cdot), Z(\cdot), v(\cdot)) \in \mathbb{R}^{n} \times \mathbb{R}^{n} \times \mathbb{R}^{n \times l} \times \mathbb{R}^{n \times d} \times \mathbb{R}^{k}, x \in \mathbb{R}^{n}$ is a given constant, and $T>0$,

$$
\begin{aligned}
& F:[0, T] \times \mathbb{R}^{n} \times \mathbb{R}^{n} \times \mathbb{R}^{n \times l} \times \mathbb{R}^{n \times d} \times \mathbb{R}^{k} \rightarrow \mathbb{R}^{n}, \\
& f:[0, T] \times \mathbb{R}^{n} \times \mathbb{R}^{n} \times \mathbb{R}^{n \times l} \times \mathbb{R}^{n \times d} \times \mathbb{R}^{k} \rightarrow \mathbb{R}^{n}, \\
& G:[0, T] \times \mathbb{R}^{n} \times \mathbb{R}^{n} \times \mathbb{R}^{n \times l} \times \mathbb{R}^{n \times d} \times \mathbb{R}^{k} \rightarrow \mathbb{R}^{n \times l}, \\
& g:[0, T] \times \mathbb{R}^{n} \times \mathbb{R}^{n} \times \mathbb{R}^{n \times l} \times \mathbb{R}^{n \times d} \times \mathbb{R}^{k} \rightarrow \mathbb{R}^{n \times d}, \\
& \Phi: \mathbb{R}^{n} \rightarrow \mathbb{R}^{n} .
\end{aligned}
$$

We assume that the state processes $(y(\cdot), Y(\cdot), z(\cdot), Z(\cdot))$ cannot be observed directly, but the controllers can observe a related noisy process $X$ of the state processes which is described by

$$
X(t)=\int_{0}^{t} h\left(s, y^{v}(s), Y^{v}(s), v(s)\right) \mathrm{d} s+\widetilde{W}(t),
$$

where $\widetilde{W}(t)$ denotes a stochastic process depending on the control variable $v(t), 0 \leq t \leq T$, and

$$
h:[0, T] \times \mathbb{R}^{n} \times \mathbb{R}^{n} \times \mathbb{R}^{k} \rightarrow \mathbb{R}^{r} .
$$


Next we will give some notations:

$$
\zeta=\left(\begin{array}{c}
y \\
Y \\
z \\
Z
\end{array}\right), \quad A(t, \zeta)=\left(\begin{array}{c}
-F \\
f \\
-G \\
g
\end{array}\right)(t, \zeta)
$$

We use the usual inner product $\langle\cdot, \cdot\rangle$ and Euclidean norm $|\cdot|$ in $\mathbb{R}^{n}, \mathbb{R}^{n \times d}$ and $\mathbb{R}^{n \times l}$.* appearing in the superscripts denotes the transpose of a matrix. All the equalities and inequalities mentioned in this paper are in the sense of $\mathrm{d} t \otimes \mathrm{d} P$ almost surely on $[0, T] \times \Omega$. We assume that

$$
\left\{\begin{array}{l}
\text { For each } \zeta \in \mathbb{R}^{n+n+n \times l+n \times d}, A(\cdot, \zeta) \text { is an } \mathcal{F}_{t} \text {-measurable process defined on } \\
{[0, T] \text { with } A(\cdot, 0) \in M^{2}\left(0, T ; \mathbb{R}^{n+n+n \times l+n \times d}\right) .}
\end{array}\right.
$$

(H2) $A(t, \zeta)$ and $\Phi(y)$ satisfy Lipschitz conditions: there exists a constant $k>0$, such that

$$
\left\{\begin{array}{l}
|A(t, \zeta)-A(t, \bar{\zeta})| \leq k|\zeta-\bar{\zeta}|, \quad \forall \zeta, \bar{\zeta} \in \mathbb{R}^{n+n+n \times l+n \times d}, \forall t \in[0, T], \\
|\Phi(y)-\Phi(\bar{y})| \leq k|y-\bar{y}|, \quad \forall y, \quad \bar{y} \in \mathbb{R}^{n} .
\end{array}\right.
$$

The following monotonic conditions introduced in [17], are main assumptions in this paper.

$$
\left\{\begin{array}{l}
\langle A(t, \zeta)-A(t, \bar{\zeta}), \zeta-\bar{\zeta}\rangle \leq-\mu|\zeta-\bar{\zeta}|^{2} \\
\quad \forall \zeta=(y, Y, z, Z)^{*}, \bar{\zeta}=(\bar{y}, \bar{Y}, \bar{z}, \bar{Z})^{*} \in \mathbb{R}^{n} \times \mathbb{R}^{n} \times \mathbb{R}^{n \times l} \times \mathbb{R}^{n \times d}, \forall t \in[0, T] \\
\langle\Phi(y)-\Phi(\bar{y}), y-\bar{y}\rangle \geq 0, \forall y, \bar{y} \in \mathbb{R}^{n}
\end{array}\right.
$$

or

$$
\left\{\begin{array}{l}
\langle A(t, \zeta)-A(t, \bar{\zeta}), \zeta-\bar{\zeta}\rangle \geq \mu|\zeta-\bar{\zeta}|^{2} \\
\quad \forall \zeta=(y, Y, z, Z)^{*}, \bar{\zeta}=(\bar{y}, \bar{Y}, \bar{z}, \bar{Z})^{*} \in \mathbb{R}^{n} \times \mathbb{R}^{n} \times \mathbb{R}^{n \times l} \times \mathbb{R}^{n \times d}, \forall t \in[0, T] \\
\langle\Phi(y)-\Phi(\bar{y}), y-\bar{y}\rangle \leq 0, \forall y, \bar{y} \in \mathbb{R}^{n}
\end{array}\right.
$$

where $\mu$ is a positive constant.

We have the following two parallel existence and uniqueness theorems.

Proposition 2.1. For any given admissible control $v(\cdot)$, we assume $(\mathrm{H} 1)$, (H2) and (H3) hold. Then FBDSDEs (2.1) has a unique solution $(y(t), Y(t), z(t), Z(t)) \in M^{2}\left(0, T ; \mathbb{R}^{n+n+n \times l+n \times d}\right)$.

Proposition 2.2. For any given admissible control $v(\cdot)$, we assume $(\mathrm{H} 1)$, $(\mathrm{H} 2)$ and $(\mathrm{H} 3)^{\prime}$ hold. Then FBDSDEs (2.1) has a unique solution $(y(t), Y(t), z(t), Z(t)) \in M^{2}\left(0, T ; \mathbb{R}^{n+n+n \times l+n \times d}\right)$.

The proof of Proposition 2.1 can be seen in [17]. For reader's convenience we give the main line of the proof of Proposition 2.1 in Appendix. The proof of Proposition 2.2 is similar, we omit it.

We assume:

$$
\left\{\begin{array}{l}
\text { (i) } F, f, G, g, \Phi \text { are continuously differentiable with respect to }(y, Y, z, Z, v), y \text { and } Y \text {, respectively; } \\
\text { (ii) the partial derivatives of } F, f, G, g, \Phi \text { are bounded; } \\
\text { (iii) } h \text { is continuously differentiable in }(y, Y, v) \text {, its derivatives and } h \text { are all uniformly bounded. }
\end{array}\right.
$$

Lastly, we need the following extension of Itô's formula (for more details see [15]). 
Proposition 2.3. Let $\alpha \in S^{2}\left([0, T] ; \mathbb{R}^{k}\right), \beta \in M^{2}\left([0, T] ; \mathbb{R}^{k}\right), \gamma \in M^{2}\left([0, T] ; \mathbb{R}^{k \times l}\right), \delta \in M^{2}\left([0, T] ; \mathbb{R}^{k \times d}\right)$ satisfy:

$$
\alpha(t)=\alpha(0)+\int_{0}^{t} \beta(s) \mathrm{d} s+\int_{0}^{t} \gamma(s) \overleftarrow{\mathrm{d}} B(s)+\int_{0}^{t} \delta(s) \overrightarrow{\mathrm{d}} W(s), \quad 0 \leq t \leq T
$$

Then

$$
\begin{aligned}
|\alpha(t)|^{2}= & |\alpha(0)|^{2}+2 \int_{0}^{t}(\alpha(s), \beta(s)) \mathrm{d} s+2 \int_{0}^{t}(\alpha(s), \gamma(s) \overleftarrow{\mathrm{d}} B(s)) \\
& +2 \int_{0}^{t}(\alpha(s), \delta(s) \overrightarrow{\mathrm{d}} W(s))-\int_{0}^{t}|\gamma(s)|^{2} \mathrm{~d} s+\int_{0}^{t}|\delta(s)|^{2} \mathrm{~d} s \\
\mathbb{E}|\alpha(t)|^{2}= & \mathbb{E}|\alpha(0)|^{2}+2 \mathbb{E} \int_{0}^{t}(\alpha(s), \beta(s)) \mathrm{d} s-\mathbb{E} \int_{0}^{t}|\gamma(s)|^{2} \mathrm{~d} s+\mathbb{E} \int_{0}^{t}|\delta(s)|^{2} \mathrm{~d} s .
\end{aligned}
$$

Here $S^{2}\left([0, T] ; \mathbb{R}^{k}\right)$ denotes the space of (classes of $\mathrm{d} P \otimes \mathrm{d} t$ a.e. equal) all $\mathcal{F}_{t}$-progressively measurable $k$-dimensional processes $v$ with

$$
\mathbb{E}\left(\sup _{0 \leq t \leq T}|v(t)|^{2}\right)<\infty
$$

Under the above hypotheses, for every $v(\cdot) \in \mathcal{U}_{a d}$, the state equation $(2.1)$ admits a unique solution $(y, Y, z, Z) \in$ $M^{2}\left(0, T ; \mathbb{R}^{n}\right) \times M^{2}\left(0, T ; \mathbb{R}^{n}\right) \times M^{2}\left(0, T ; \mathbb{R}^{n \times l}\right) \times M^{2}\left(0, T ; \mathbb{R}^{n \times d}\right)$. (see Peng and Shi $\left.[17]\right)$.

Define $\mathrm{d} P^{v} \doteq \rho^{v}(t) \mathrm{d} P$, where

$$
\rho^{v}(t) \doteq \exp \left\{\int_{0}^{t}\left\langle h\left(s, y^{v}(s), Y^{v}(s), v(s)\right), \overrightarrow{\mathrm{d}} X(s)\right\rangle-\frac{1}{2} \int_{0}^{t}\left|h\left(s, y^{v}(s), Y^{v}(s), v(s)\right)\right|^{2} \mathrm{~d} s\right\} .
$$

Obviously, $\rho^{v}(t) \in \mathbb{R}$ is the solution to the following stochastic differential equation (SDE in short):

$$
\left\{\begin{array}{l}
\mathrm{d} \rho^{v}(t)=\rho^{v}(t)\left\langle h\left(t, y^{v}(t), Y^{v}(t), v(t)\right), \overrightarrow{\mathrm{d}} X(t)\right\rangle, \\
\rho^{v}(0)=1
\end{array}\right.
$$

Hence by Girsanov's theorem and (H4), $P^{v}$ is a new probability measure and $(W(\cdot), \widetilde{W}(\cdot), B(\cdot))$ is a $\mathbb{R}^{l+d+r_{-}}$ valued standard Brownian motion defined on the new probability space $\left(\Omega, \mathcal{F}, P^{v}\right)$. Note that the integrals with respect to $\{X(t)\}$ and $\{\widetilde{W}(t)\}$ are standard forward Itô integrals.

We introduce the following cost functional:

$$
J(v(\cdot))=\mathbb{E}^{v}\left\{\int_{0}^{T} l\left(t, y^{v}(t), Y^{v}(t), z^{v}(t), Z^{v}(t), v(t)\right) \mathrm{d} t+\psi\left(y^{v}(T)\right)+\gamma\left(Y^{v}(0)\right)\right\},
$$

where $\mathbb{E}^{v}$ denotes the expectation with respect to the probability space $\left(\Omega, \mathcal{F}, P^{v}\right)$, and

$$
\begin{aligned}
& l:[0, T] \times \mathbb{R}^{n} \times \mathbb{R}^{n} \times \mathbb{R}^{n \times l} \times \mathbb{R}^{n \times d} \times \mathbb{R}^{k} \rightarrow \mathbb{R}, \\
& \psi: \mathbb{R}^{n} \rightarrow \mathbb{R}, \\
& \gamma: \mathbb{R}^{n} \rightarrow \mathbb{R} .
\end{aligned}
$$

We also assume

\footnotetext{
(i) $l$ is continuously differentiable in $(y, Y, z, Z, v)$, its partial derivatives are continuous in $(y, Y, z, Z, v)$ $\{$ and bounded by $c(1+|y|+|Y|+|z|+|Z|+|v|)$;

(ii) the derivatives of $\psi$ and $\gamma$ with respect to $y, Y$ are bounded by $C(1+|y|)$ and $C(1+|Y|)$, respectively.
} 
Our partially-observed optimal control problem is to minimize the cost functional (2.4) over $v(\cdot) \in \mathcal{U}_{a d}$ subject to $(2.1)$ and $(2.2)$, i.e., to find $u(\cdot) \in \mathcal{U}_{a d}$ satisfying

$$
J(u(\cdot))=\min _{v(\cdot) \in \mathcal{U}_{a d}} J(v(\cdot)) .
$$

Obviously, cost functional (2.4) can be rewritten as

$$
J(v(\cdot))=\mathbb{E}\left\{\int_{0}^{T} \rho^{v}(t) l\left(t, y^{v}(t), Y^{v}(t), z^{v}(t), Z^{v}(t), v(t)\right) \mathrm{d} t+\rho^{v}(T) \psi\left(y^{v}(T)\right)+\gamma\left(Y^{v}(0)\right)\right\} .
$$

Then the original problem (2.5) is equivalent to minimizing (2.6) over $v(\cdot) \in \mathcal{U}_{a d}$ subject to (2.1) and (2.3). Our main target is to find the necessary condition of the partially-observed optimal control $u(\cdot)$ in the form of Pontryagin stochastic maximum principle.

\section{Partially-observed stochastic maximum PRinciple}

For the convex admissible control set, the classical way to derive necessary optimality conditions is to use the convex perturbation method. Let $u(\cdot)$ be an optimal control and $(y(\cdot), Y(\cdot), z(\cdot), Z(\cdot))$ be the corresponding optimal trajectory. Let $v(\cdot)$ be such that $u(\cdot)+v(\cdot) \in \mathcal{U}_{a d}$. Since $\mathcal{U}_{a d}$ is convex, then for any $0 \leq \varepsilon \leq 1$, $u^{\varepsilon}(\cdot)=u(\cdot)+\varepsilon v(\cdot)$ is also in $\mathcal{U}_{a d}$. We denote the solution of (2.1) and (2.3) corresponding to the control $u^{\varepsilon}(\cdot)$ by $\left(y^{\varepsilon}(\cdot), Y^{\varepsilon}(\cdot), z^{\varepsilon}(\cdot), Z^{\varepsilon}(\cdot)\right)$ and $\rho^{\varepsilon}(\cdot)$.

For convenience, we introduce the notations

$$
\begin{aligned}
\varphi(t) & =\varphi(t, y(t), Y(t), z(t), Z(t), u(t)), \\
\varphi^{\varepsilon}(t) & =\varphi\left(t, y^{\varepsilon}(t), Y^{\varepsilon}(t), z^{\varepsilon}(t), Z^{\varepsilon}(t), u(t)+\varepsilon v(t)\right),
\end{aligned}
$$

where $\varphi$ denotes one of $F, f, G, g, l$, and their partial derivatives.

We introduce the variational equations consisting of a FBDSDE and another forward SDE as follows:

$$
\left\{\begin{aligned}
\mathrm{d} y^{1}(t)= & {\left[f_{y}(t) y^{1}(t)+f_{Y}(t) Y^{1}(t)+f_{z}(t) z^{1}(t)+f_{Z}(t) Z^{1}(t)+f_{v}(t) v(t)\right] \mathrm{d} t } \\
& +\left[g_{y}(t) y^{1}(t)+g_{Y}(t) Y^{1}(t)+g_{z}(t) z^{1}(t)+g_{Z}(t) Z^{1}(t)+g_{v}(t) v(t)\right] \overrightarrow{\mathrm{d}} W(t)-z^{1}(t) \overleftarrow{\mathrm{d}} B(t), \\
\mathrm{d} Y^{1}(t)= & -\left[F_{y}(t) y^{1}(t)+F_{Y}(t) Y^{1}(t)+F_{z}(t) z^{1}(t)+F_{Z}(t) Z^{1}(t)+F_{v}(t) v(t)\right] \mathrm{d} t \\
& -\left[G_{y}(t) y^{1}(t)+G_{Y}(t) Y^{1}(t)+G_{z}(t) z^{1}(t)+G_{Z}(t) Z^{1}(t)+G_{v}(t) v(t)\right] \mathrm{d} B(t)+Z^{1}(t) \overrightarrow{\mathrm{d}} W(t) \\
y^{1}(0)= & 0, \quad Y^{1}(T)=\Phi_{y}(y(T)) y^{1}(T),
\end{aligned}\right.
$$

and

$$
\left\{\begin{array}{l}
\mathrm{d} \rho^{1}(t)=\left[\rho^{1}(t) h^{*}(t)+\rho(t)\left(h_{y}(t) y^{1}(t)\right)^{*}+\rho(t)\left(h_{Y}(t) Y^{1}(t)\right)^{*}+\rho(t)\left(h_{v}(t) v(t)\right)^{*}\right] \overrightarrow{\mathrm{d}} X(t) \\
\rho^{1}(0)=0
\end{array}\right.
$$

By (H4), it is easy to see that (3.1) and (3.2) admit unique measurable solutions $\left(y^{1}(t), Y^{1}(t), z^{1}(t), Z^{1}(t)\right) \in$ $M^{2}\left(0, T ; \mathbb{R}^{n}\right) \times M^{2}\left(0, T ; \mathbb{R}^{n}\right) \times M^{2}\left(0, T ; \mathbb{R}^{n \times l}\right) \times M^{2}\left(0, T ; \mathbb{R}^{n \times d}\right)$ and $\rho^{1}(t) \in M_{\mathcal{F} X}^{2}(0, T ; \mathbb{R})$, respectively.

By Lemma 4 in [29] and Lemma 3.1 in [32], we can get the solutions depending on parameter to forwardbackward doubly stochastic control system. 
Lemma 3.1. Assume that (H1)-(H4) hold. Then we have

$$
\begin{aligned}
& \lim _{\varepsilon \rightarrow 0} \frac{y^{\varepsilon}(t)-y(t)}{\varepsilon}=y^{1}(t), \\
& \lim _{\varepsilon \rightarrow 0} \frac{Y^{\varepsilon}(t)-Y(t)}{\varepsilon}=Y^{1}(t), \\
& \lim _{\varepsilon \rightarrow 0} \frac{z^{\varepsilon}(t)-z(t)}{\varepsilon}=z^{1}(t), \\
& \lim _{\varepsilon \rightarrow 0} \frac{Z^{\varepsilon}(t)-Z(t)}{\varepsilon}=Z^{1}(t), \\
& \lim _{\varepsilon \rightarrow 0} \frac{\rho^{\varepsilon}(t)-\rho(t)}{\varepsilon}=\rho^{1}(t) .
\end{aligned}
$$

Since $u(\cdot)$ is an optimal control, then

$$
\varepsilon^{-1}\left[J\left(u^{\varepsilon}(\cdot)\right)-J(u(\cdot)] \geq 0 .\right.
$$

From this and Lemma 3.1, we have the following

Lemma 3.2. Let assumptions (H1)-(H4) hold. Then the following variational inequality holds:

$$
\begin{aligned}
\mathbb{E}^{u} \int_{0}^{T}\left[\rho^{-1}(t) \rho^{1}(t) l(t)+l_{y}^{*}(t)\right. & \left.y^{1}(t)+l_{Y}^{*}(t) Y^{1}(t)+l_{z}^{*}(t) z^{1}(t)+l_{Z}^{*}(t) Z^{1}(t)+l_{v}^{*}(t) v(t)\right] \mathrm{d} t \\
& +\mathbb{E}^{u}\left[\psi_{y}^{*}(y(T)) y^{1}(T)\right]+\mathbb{E}^{u}\left[\gamma_{Y}^{*}(Y(0)) Y^{1}(0)\right]+\mathbb{E}^{u}\left[\rho^{-1}(T) \rho^{1}(T) \psi(y(T))\right] \geq 0 .
\end{aligned}
$$

Proof. Let $\varepsilon \rightarrow 0$ in (3.3). From Lemma 3.1, we derive

$$
\begin{array}{r}
\varepsilon^{-1}\left\{\mathbb{E} \int_{0}^{T}\left[\rho^{\varepsilon}(t) l^{\varepsilon}(t)-\rho(t) l(t)\right] \mathrm{d} t\right\} \rightarrow \mathbb{E} \int_{0}^{T}\left[\rho^{1}(t) l(t)+\rho(t) l_{y}^{*}(t) y^{1}(t)+\rho(t) l_{Y}^{*}(t) Y^{1}(t)\right. \\
\left.+\rho(t) l_{z}^{*}(t) z^{1}(t)+\rho(t) l_{Z}^{*}(t) Z^{1}(t)+\rho(t) l_{v}^{*}(t) v(t)\right] \mathrm{d} t
\end{array}
$$

Similarly, we have

$$
\begin{aligned}
\varepsilon^{-1} \mathbb{E}\left[\gamma\left(Y^{\varepsilon}(0)\right)-\gamma(Y(0))\right] & \rightarrow \mathbb{E}\left[\gamma_{Y}^{*}(Y(0)) Y^{1}(0)\right] \\
\varepsilon^{-1} \mathbb{E}\left[\rho^{\varepsilon}(T) \psi\left(y^{\varepsilon}(T)\right)-\rho(T) \psi(y(T))\right] & \rightarrow \mathbb{E}\left[\rho(T) \psi_{y}^{*}(y(T)) y^{1}(T)\right]+\mathbb{E}\left[\rho^{1}(T) \psi(y(T))\right]
\end{aligned}
$$

Then, we have

$$
\begin{aligned}
\mathbb{E} \int_{0}^{T}\left[\rho^{1}(t) l(t)+\rho(t) l_{y}^{*}(t) y^{1}(t)+\rho(t) l_{Y}^{*}(t) Y^{1}(t)+\rho(t) l_{z}^{*}(t) z^{1}(t)+\rho(t) l_{Z}^{*}(t) Z^{1}(t)+\rho(t) l_{v}^{*}(t) v(t)\right] \mathrm{d} t \\
+\mathbb{E}\left[\gamma_{Y}^{*}(Y(0)) Y^{1}(0)\right]+\mathbb{E}\left[\rho(T) \psi_{y}^{*}(y(T)) y^{1}(T)\right]+\mathbb{E}\left[\rho^{1}(T) \psi(y(T))\right] \geq 0 .
\end{aligned}
$$

Thus (3.4) follows. The proof of Lemma 3.2 is completed.

Noticing the term $\rho^{-1}(t) \rho^{1}(t)$ in the above variational inequality (3.4), we let $\Gamma(t)=\rho^{-1}(t) \rho^{1}(t)$ and from equations (2.2), (2.3) and (3.2), for the optimal control $u(\cdot)$, we have

$$
\left\{\begin{array}{l}
\mathrm{d} \Gamma(t)=\left[h_{y}(t, y, Y, u) y^{1}(t)+h_{Y}(t, y, Y, u) Y^{1}(t)+h_{v}(t, y, Y, u) v(t)\right]^{*} \overrightarrow{\mathrm{d}} \widetilde{W}(t), \\
\Gamma(0)=0
\end{array}\right.
$$


To derive the maximum principle, we first introduce the adjoint equation corresponding to (3.5), which is an auxiliary BSDE and whose solution is denoted by $(P(\cdot), Q(\cdot)) \in M_{\mathcal{F}^{X}}^{2}(0, T ; \mathbb{R}) \times M_{\mathcal{F} X}^{2}\left(0, T ; \mathbb{R}^{n}\right)$,

$$
\left\{\begin{array}{l}
-\mathrm{d} P(t)=l(t) \mathrm{d} t-Q(t) \overrightarrow{\mathrm{d}} \widetilde{W}(t), \\
P(T)=\psi(y(T)) .
\end{array}\right.
$$

Next, we introduce the adjoint equation corresponding to variational equation (3.4), which is a FBDSDE and whose solution is denoted by $(p(\cdot), q(\cdot), k(\cdot), m(\cdot)) \in M^{2}\left(0, T ; \mathbb{R}^{n}\right) \times M^{2}\left(0, T ; \mathbb{R}^{n}\right) \times M^{2}\left(0, T ; \mathbb{R}^{n \times l}\right) \times$ $M^{2}\left(0, T ; \mathbb{R}^{n \times d}\right)$,

$$
\left\{\begin{aligned}
\mathrm{d} p(t)= & {\left[F_{Y}^{*}(t) p(t)-f_{Y}^{*}(t) q(t)+G_{Y}^{*}(t) k(t)-g_{Y}^{*}(t) m(t)-h_{Y}^{*}(t) Q(t)-l_{Y}(t)\right] \mathrm{d} t } \\
& +\left[F_{Z}^{*}(t) p(t)-f_{Z}^{*}(t) q(t)+G_{Z}^{*}(t) k(t)-g_{Z}^{*}(t) m(t)-l_{Z}(t)\right] \overrightarrow{\mathrm{d}} W(t)-k(t) \overleftarrow{\mathrm{d}} B(t) \\
\mathrm{d} q(t)= & {\left[F_{y}^{*}(t) p(t)-f_{y}^{*}(t) q(t)+G_{y}^{*}(t) k(t)-g_{y}^{*}(t) m(t)-h_{y}^{*}(t) Q(t)-l_{y}(t)\right] \mathrm{d} t } \\
& +\left[F_{z}^{*}(t) p(t)-f_{z}^{*}(t) q(t)+G_{z}^{*}(t) k(t)-g_{z}^{*}(t) m(t)-l_{z}(t)\right] \overleftarrow{\mathrm{d}} B(t)+m(t) \overrightarrow{\mathrm{d}} W(t), \\
p(0)= & -\gamma_{Y}(Y(0)), \quad q(T)=-\Phi_{y}^{*}(y(T)) p(T)+\psi_{y}(y(T)) .
\end{aligned}\right.
$$

Under (H1)-(H4), by the result on forward-backward doubly stochastic differential equations (see Peng and Shi [17]), we can show that equation (3.7) admits a unique solution $(p(t), q(t), k(t), m(t)) \in M^{2}\left(0, T ; \mathbb{R}^{n}\right) \times$ $M^{2}\left(0, T ; \mathbb{R}^{n}\right) \times M^{2}\left(0, T ; \mathbb{R}^{n \times l}\right) \times M^{2}\left(0, T ; \mathbb{R}^{n \times d}\right)$.

We define the Hamiltonian function $H:[0, T] \times \mathbb{R}^{n} \times \mathbb{R}^{n} \times \mathbb{R}^{n \times l} \times \mathbb{R}^{n \times d} \times \mathbb{R}^{k} \times \mathbb{R}^{n} \times \mathbb{R}^{n} \times \mathbb{R}^{n \times l} \times \mathbb{R}^{n \times d} \times \mathbb{R}^{r} \rightarrow \mathbb{R}$ as follows:

$$
\begin{aligned}
H(t, y, Y, z, Z, v, p, q, k, m, Q)= & \langle q(t), f(t, y, Y, z, Z, v)\rangle-\langle p(t), F(t, y, Y, z, Z, v)\rangle+\langle m(t), g(t, y, Y, z, Z, v)\rangle \\
& -\langle k(t), G(t, y, Y, z, Z, v)\rangle+\langle Q(t), h(t, y, Y, v)\rangle+l(t, y, Y, z, Z, v) .
\end{aligned}
$$

Denote $H(t) \equiv H(t, y(t), Y(t), z(t), Z(t), v(t), p(t), q(t), k(t), m(t), Q(t))$ and its derivatives, then adjoint equations (3.7) can be rewritten as the following stochastic Hamiltonian system's type FBDSDE and whose solution is denoted by $(p(\cdot), q(\cdot), k(\cdot), m(\cdot))$,

$$
\left\{\begin{array}{l}
\mathrm{d} p(t)=-H_{Y}(t) \mathrm{d} t-H_{Z}(t) \overrightarrow{\mathrm{d}} W(t)-k(t) \overleftarrow{\mathrm{d}} B(t) \\
\mathrm{d} q(t)=-H_{y}(t) \mathrm{d} t-H_{z}(t) \overleftarrow{\mathrm{d}} B(t)+m(t) \overrightarrow{\mathrm{d}} W(t) \\
p(0)=-\gamma_{Y}(Y(0)), \quad q(T)=-\Phi_{y}(y(T)) p(T)+\psi_{y}(y(T))
\end{array}\right.
$$

Starting from the variational inequality (3.4), we can now state the main result of this paper.

Theorem 3.1 (Partially-observed stochastic maximum principle). Suppose (H1)-(H4) hold. Let $u(\cdot)$ be an optimal control for our partially-observed optimal control problem $(2.5),(y(\cdot), Y(\cdot), z(\cdot), Z(\cdot))$ be the optimal trajectory and $\rho(\cdot)$ be the corresponding solution of $(2.3)$. Let $(P(\cdot), Q(\cdot))$ be the solution of (3.6) and $(p(\cdot), q(\cdot), k(\cdot), m(\cdot))$ be the solution of adjoint equation (3.7). Then the following maximum principle

$$
\begin{aligned}
\mathbb{E}^{u}\left[\left\langle H_{v}(t, y(t), Y(t), z(t), Z(t), u(t), p(t), q(t), k(t), m(t), Q(t)), v-u(t)\right\rangle \mid \mathcal{F}_{t}^{X}\right] \geq 0, & \\
\forall v & \in \text {, a.e. } t \in[0, T], \text { a.s. }
\end{aligned}
$$

holds, where the Hamiltonian function $H$ is defined by (3.8). 
Proof. Applying Itô's formula to $\left\langle y^{1}(t), q(t)\right\rangle+\left\langle Y^{1}(t), p(t)\right\rangle+\langle\Gamma(t), P(t)\rangle$. By the variational equations (3.1), (3.2), variational inequality (3.4), auxiliary BSDE (3.6) and adjoint equation (3.7), we obtain

$$
\begin{aligned}
& \mathbb{E}^{u} \int_{0}^{T}\left[\Gamma(t) l(t)+l_{y}^{*}(t) y^{1}(t)+l_{Y}^{*}(t) Y^{1}(t)+l_{z}^{*}(t) z^{1}(t)+l_{Z}^{*}(t) Z^{1}(t)+l_{v}^{*}(t) v(t)\right] \mathrm{d} t \\
& +\mathbb{E}^{u}\left[\psi_{y}^{*}(y(T)) y^{1}(T)\right]+\mathbb{E}^{u}\left[\gamma_{Y}^{*}(Y(0)) Y^{1}(0)\right]+\mathbb{E}^{u}\left[\rho^{-1}(T) \rho^{1}(T) \psi(y(T))\right] \\
= & \mathbb{E}^{u} \int_{0}^{T}\left\langle f_{v}^{*}(t) q(t)-F_{v}^{*}(t) p(t)+g_{v}^{*}(t) k(t)-G_{v}^{*}(t) m(t)+h_{v}^{*}(t) Q(t)+l_{v}(t), v(t)\right\rangle \mathrm{d} t \\
= & \mathbb{E}^{u} \int_{0}^{T}\left\langle H_{v}(t, y(t), Y(t), z(t), Z(t), u(t), p(t), q(t), k(t), m(t), Q(t)), v(t)\right\rangle \mathrm{d} t \geq 0 .
\end{aligned}
$$

Because $v(t)$ satisfies $u(t)+v(t) \in U$, we have

$$
\mathbb{E}^{u} \int_{0}^{T}\left\langle H_{v}(t, y(t), Y(t), z(t), Z(t), u(t), p(t), q(t), k(t), m(t), Q(t)), v-u(t)\right\rangle \mathrm{d} t \geq 0, \forall v \in U
$$

This implies that

$$
\mathbb{E}^{u}\left\langle H_{v}(t, y(t), Y(t), z(t), Z(t), u(t), p(t), q(t), k(t), m(t), Q(t)), v-u(t)\right\rangle \geq 0, \forall v \in U .
$$

Now, let $v \in U$ be a deterministic element and $F$ be an arbitrary element of the $\sigma$-algebra $\mathcal{F}_{t}^{X}$. And set

$$
w(t)=v \mathbf{1}_{F}+u(t) \mathbf{1}_{\Omega-F} .
$$

It is obvious that $w$ is an admissible control.

Applying the above inequality with $w$, we get

$$
\begin{aligned}
& \mathbb{E}^{u}\left[\mathbf{1}_{F}\left\langle H_{v}(t, y(t), Y(t), z(t), Z(t), u(t), p(t), q(t), k(t), m(t), Q(t)), v-u(t)\right\rangle\right] \geq 0, \\
& \forall F \in \mathcal{F}_{t}^{X},
\end{aligned}
$$

which implies that

$$
\begin{aligned}
& \mathbb{E}^{u}\left[\left\langle H_{v}(t, y(t), Y(t), z(t), Z(t), u(t), p(t), q(t), k(t), m(t), Q(t)), v-u(t)\right\rangle \mid \mathcal{F}_{t}^{X}\right] \geq 0, \\
& \forall v \in U, \text { a.e. } t \in[0, T], \text { a.s. }
\end{aligned}
$$

The proof of Theorem 3.1 is completed.

\section{Applications in LQ control problems}

In this section, we give an example to show applications of the main results in this paper. We desire to apply our maximal principle to forward-backward doubly stochastic LQ control problems. For notational simplification, we assume $d=l=1$ and $U=\mathbb{R}$. Let us consider the following:

$$
\left\{\begin{aligned}
\mathrm{d} y^{v}(t)= & {\left[A_{1}(t) y^{v}(t)-b Y^{v}(t)+A_{2}(t) z^{v}(t)+B_{1}(t) v(t)\right] \mathrm{d} t-z^{v}(t) \overleftarrow{\mathrm{d}} B(t) } \\
& +\left[A_{3}(t) y^{v}(t)+B_{2}(t) v(t)\right] \overrightarrow{\mathrm{d}} W(t) \\
\mathrm{d} Y^{v}(t)= & -\left[a y^{v}(t)+A_{1}(t) Y^{v}(t)+A_{3}(t) Z^{v}(t)+B_{3}(t) v(t)\right] \mathrm{d} t+Z^{v}(t) \overrightarrow{\mathrm{d}} W(t) \\
& -\left[A_{2}(t) Y^{v}(t)+B_{4}(t) v(t)\right] \overleftarrow{\mathrm{d}} B(t) \\
y^{v}(0)= & x, \quad Y^{v}(T)=c y^{v}(T)
\end{aligned}\right.
$$


and observation

$$
\mathrm{d} X(t)=F(t) \mathrm{d} t+\overrightarrow{\mathrm{d}} \widetilde{W}(t), t \in[0, T], X(0)=0 .
$$

The cost functional is

$$
J(v(\cdot))=\frac{1}{2} \mathbb{E}^{v}\left[\int_{0}^{T} M(t) v^{2}(t) \mathrm{d} t+R_{T}\left(y^{v}(T)\right)^{2}+S_{0}\left(Y^{v}(0)\right)^{2}\right],
$$

where constants $a>0, b>0, c>0, M(t)>0, R_{T} \geq 0$ and $S_{0} \geq 0$. Functions $A_{i}(\cdot), i=1,2,3$, and $B_{i}(\cdot), i=1,2,3,4$, are bounded and deterministic, $M^{-1}(\cdot)$ is also bounded. $W(\cdot), B(\cdot), X(\cdot)$ are three mutually independent standard Brownian motions on $(\Omega, \mathcal{F}, P)$.

For any given $v(\cdot)$, it is easy to show that the monotonic condition (H3) holds. Then FBDSDE (4.1) admits a unique solution $(y(\cdot), Y(\cdot), z(\cdot), Z(\cdot))$. By $(3.8)$, the Hamiltonian function is given by

$$
\begin{aligned}
H(t, y, Y, z, Z, v, p, q, k, m)= & q(t)\left[A_{1}(t) y^{v}(t)-b Y^{v}(t)+A_{2}(t) z^{v}(t)+B_{1}(t) v(t)\right] \\
& -p(t)\left[a y^{v}(t)+A_{1}(t) Y^{v}(t)+A_{3}(t) Z^{v}(t)+B_{3}(t) v(t)\right] \\
& -k(t)\left[A_{2}(t) Y^{v}(t)+B_{4}(t) v(t)\right]+m(t)\left[A_{3}(t) y^{v}(t)+B_{2}(t) v(t)\right] \\
& +Q(t) F(t)+\frac{1}{2} M(t) v^{2}(t) .
\end{aligned}
$$

According to Theorem 3.1, if $u(\cdot)$ is optimal, then

$$
u(t)=-M^{-1}(t)\left(B_{1}(t) \mathbb{E}^{u}\left[q(t) \mid \mathcal{F}_{t}^{X}\right]-B_{3}(t) \mathbb{E}^{u}\left[p(t) \mid \mathcal{F}_{t}^{X}\right]+B_{2}(t) \mathbb{E}^{u}\left[m(t) \mid \mathcal{F}_{t}^{X}\right]-B_{4}(t) \mathbb{E}^{u}\left[k(t) \mid \mathcal{F}_{t}^{X}\right]\right) .
$$

where $(p(\cdot), q(\cdot), k(\cdot), m(\cdot))$ is the solution of the following FBDSDE:

$$
\left\{\begin{array}{l}
\mathrm{d} p(t)=\left[b q(t)+A_{1}(t) p(t)+A_{2}(t) k(t)\right] \mathrm{d} t+A_{3}(t) p(t) \overrightarrow{\mathrm{d}} W(t)-k(t) \overleftarrow{\mathrm{d}} B(t), \\
\mathrm{d} q(t)=-\left[A_{1}(t) q(t)-a p(t)+A_{3}(t) m(t)\right] \mathrm{d} t-A_{2}(t) q(t) \overleftarrow{\mathrm{d}} B(t)-m(t) \overrightarrow{\mathrm{d}} W(t), \\
p(0)=-R_{0} Y^{u}(0), \quad q(T)=-c p(T)+S_{T} y^{u}(T) .
\end{array}\right.
$$

Similarly, it is easy to verify that the monotonic condition (H3) holds, then FBDSDE (4.6) admits a unique solution $(p(\cdot), q(\cdot), k(\cdot), m(\cdot))$.

Moreover, we can prove that the admissible control (4.5) which satisfying the necessary condition of optimality is really optimal. For any admissible control $v(\cdot)$, we have

$$
\begin{aligned}
& J(v(\cdot))-J(u(\cdot))=\frac{1}{2} \mathbb{E}^{u}\left\{\int_{0}^{T}\left[M(t)(v(t)-u(t))^{2}+2 M(t) u(t)(v(t)-u(t))\right] \mathrm{d} t\right. \\
& \left.\quad+S_{T}\left(y^{v}(T)-y^{u}(T)\right)^{2}+2 S_{T} y^{u}(T)\left(y^{v}(T)-y^{u}(T)\right)+R_{0}\left(Y^{v}(0)-Y^{u}(0)\right)^{2}+2 R_{0} Y^{u}(0)\left(Y^{v}(0)-Y^{u}(0)\right)\right\} .
\end{aligned}
$$

Applying Itô's formula to $\left(y^{v}(t)-y^{u}(t)\right) q(t)+\left(Y^{v}(t)-Y^{u}(t)\right) p(t)$, noting that (4.1), (4.6), we have

$$
\begin{aligned}
\mathbb{E}^{u}\left\{S_{T} y^{v}(T)\left(y^{v}(T)-y^{u}(T)\right)+R_{0} Y^{v}(0)\right. & \left.\left(Y^{v}(0)-Y^{u}(0)\right)\right\}= \\
& \mathbb{E}^{u} \int_{0}^{T}\left[\left(B_{1}(t) q(t)-B_{3}(t) p(t)+B_{2}(t) h(t)-B_{4}(t) k(t)\right)(v(t)-u(t))\right] \mathrm{d} t .
\end{aligned}
$$


As $M(t)>0, \forall t \in[0, T], S_{T} \geq 0, R_{0} \geq 0$, noting that $(p(\cdot), q(\cdot), k(\cdot), m(\cdot))$ are not observable, we have

$$
\begin{aligned}
J(v(\cdot))-J(u(\cdot)) \geq & \mathbb{E}^{u}\left\{\int_{0}^{T}[M(t) u(t)(v(t)-u(t))] \mathrm{d} t+S_{T} y^{u}(T)\left(y^{v}(T)-y^{u}(T)\right)\right. \\
& \left.+R_{0} Y^{u}(0)\left(Y^{v}(0)-Y^{u}(0)\right)\right\} \\
= & \mathbb{E}^{u} \int_{0}^{T}\left[\left(M(t) u(t)+B_{1}(t) q(t)-B_{3}(t) p(t)+B_{2}(t) m(t)-B_{4}(t) k(t)\right)(v(t)-u(t))\right] \mathrm{d} t \\
= & \mathbb{E}^{u} \int_{0}^{T}\left[\left(-M(t) M^{-1}(t)\left(B_{1}(t) \mathbb{E}^{u}\left[q(t) \mid \mathcal{F}_{t}^{X}\right]-B_{3}(t) \mathbb{E}^{u}\left[p(t) \mid \mathcal{F}_{t}^{X}\right]+B_{2}(t) \mathbb{E}^{u}\left[m(t) \mid \mathcal{F}_{t}^{X}\right]\right.\right.\right. \\
& \left.\left.\left.-B_{4}(t) \mathbb{E}^{u}\left[k(t) \mid \mathcal{F}_{t}^{X}\right]\right)+B_{1}(t) q(t)-B_{3}(t) p(t)+B_{2}(t) m(t)-B_{4}(t) k(t)\right)(v(t)-u(t))\right] \mathrm{d} t \\
= & 0 .
\end{aligned}
$$

So (4.5) is an optimal control.

Remark 4.1. Both the forward equation and the backward one in forward-backward doubly stochastic system (4.1) are types of BDSDE (1.1) with different directions of stochastic integrals. So (4.1) includes backward doubly stochastic LQ control problems and provides a very general framework of fully coupled forward-backward stochastic systems.

\section{Appendix: The proof of Proposition 2.1}

In order to prove the existence and uniqueness result for (2.1) under (H1)-(H3), we need the following lemmas. They involve a priori estimates of solutions of the following family of FBDSDEs parametrized by $\alpha \in[0,1]$.

$$
\left\{\begin{array}{l}
\mathrm{d} y(t)=\left[f^{\alpha}(t, \zeta(t))+f_{0}(t)\right] \mathrm{d} t+\left[g^{\alpha}(t, \zeta(t))+g_{0}(t)\right] \overrightarrow{\mathrm{d}} W(t)-z(t) \overleftarrow{\mathrm{d}} B(t), \\
\mathrm{d} Y(t)=\left[F^{\alpha}(t, \zeta(t))+F_{0}(t)\right] \mathrm{d} t+\left[G^{\alpha}(t, \zeta(t))+G_{0}(t)\right] \overleftarrow{\mathrm{d}} B(t)+Z(t) \overrightarrow{\mathrm{d}} W(t), \\
y(0)=x, \quad Y(T)=\Phi^{\alpha}(y(T))+\varphi
\end{array}\right.
$$

where $\zeta=(y, Y, z, Z)$ and for any given $\alpha \in \mathbb{R}$,

$$
\begin{aligned}
f^{\alpha}(t, y, Y, z, Z) & =\alpha f(t, y, Y, z, Z)-(1-\alpha) Y, \\
g^{\alpha}(t, y, Y, z, Z) & =\alpha g(t, y, Y, z, Z)-(1-\alpha) Z, \\
F^{\alpha}(t, y, Y, z, Z) & =\alpha F(t, y, Y, z, Z)-(1-\alpha) y, \\
G^{\alpha}(t, y, Y, z, Z) & =\alpha G(t, y, Y, z, Z)-(1-\alpha) z, \\
\Phi^{\alpha}(y) & =\alpha \Phi(y)+(1-\alpha) y .
\end{aligned}
$$

Observe that when $\alpha=0$, (A.1) is written in the following simple form

$$
\left\{\begin{array}{l}
\mathrm{d} y(t)=\left(-Y(t)+f_{0}(t)\right) \mathrm{d} t+\left(-Z(t)+g_{0}(t)\right) \overrightarrow{\mathrm{d}} W(t)-z(t) \overleftarrow{\mathrm{d}} B(t) \\
\mathrm{d} Y(t)=\left(-y(t)+F_{0}(t)\right) \mathrm{d} t+\left(-z(t)+G_{0}(t)\right) \overleftarrow{\mathrm{d}} B(t)+Z(t) \overrightarrow{\mathrm{d}} W(t) \\
y(0)=x, \quad Y(T)=y(T)+\varphi
\end{array}\right.
$$

We have the following lemma. 
Lemma A.1. For any $x \in \mathbb{R}^{n},\left(F_{0}, f_{0}, G_{0}, g_{0}\right) \in M^{2}\left(0, T ; \mathbb{R}^{n+n+n \times l+n \times d}\right), \varphi \in L^{2}\left(\Omega, \mathcal{F}_{T} ; \mathbb{R}^{n}\right)$, (A.2) has a unique solution $(y, Y, z, Z)$ in $M^{2}\left(0, T ; \mathbb{R}^{n+n+n \times l+n \times d}\right)$.

Proof. The proof of uniqueness is similar to the one of Proposition 2.1 below. We only need to find a solution for (A.2). We consider the following linear backward doubly stochastic differential equations

$$
\bar{Y}(t)=\varphi-\int_{t}^{T}\left[\bar{Y}(s)+F_{0}(s)-f_{0}(s)\right] \mathrm{d} s-\int_{t}^{T}\left[2 \bar{Z}(s)-g_{0}(s)\right] \overrightarrow{\mathrm{d}} W(s)-\int_{t}^{T} G_{0}(s) \overleftarrow{\mathrm{d}} B(s)
$$

By the result of [15], the above equation has a unique solution $(\bar{Y}, \bar{Z})$. Then we can solve the following SDE

$$
y(t)=x+\int_{0}^{t}\left[-y(s)-\bar{Y}(s)+f_{0}(s)\right] \mathrm{d} s+\int_{0}^{t}\left[-\bar{Z}(s)+g_{0}(s)\right] \overrightarrow{\mathrm{d}} W(s)-\int_{0}^{t} \bar{z}(s) \overleftarrow{\mathrm{d}} B(s)
$$

Due to the result in [15], the above equation has a unique solution $(y, \bar{z})$. And setting $Y=y+\bar{Y}, Z=\bar{Z}$, and $z=\bar{z}$, we easily see that $(y, Y, z, Z)$ is a solution to (A.2). Thus the existence is proven.

The following a priori lemma is a key step in the proof of the method of continuation. It shows that, if for a fixed $\alpha=\alpha_{0} \in[0,1]$, (A.1) can be solved, then it can also be solved for $\alpha \in\left[\alpha_{0}, \alpha_{0}+\delta_{0}\right]$, for some positive constant $\delta_{0}$ independent of $\alpha_{0}$.

Lemma A.2. Under assumptions (H1)-(H3), there exists a positive constant $\delta_{0}$ such that if, a priori, for some $\alpha_{0} \in[0,1)$, and for each $x \in \mathbb{R}^{n}, \varphi \in L^{2}\left(\Omega, \mathcal{F}_{T} ; \mathbb{R}^{n}\right),\left(F_{0}, f_{0}, G_{0}, g_{0}\right) \in M^{2}\left(0, T ; \mathbb{R}^{n+n+n \times l+n \times d}\right)$, (A.1) has a unique solution, then for each $\alpha \in\left[\alpha_{0}, \alpha_{0}+\delta_{0}\right]$, and $x \in \mathbb{R}^{n}, \varphi \in L^{2}\left(\Omega, \mathcal{F}_{T} ; \mathbb{R}^{n}\right),\left(F_{0}, f_{0}, G_{0}, g_{0}\right) \in$ $M^{2}\left(0, T ; \mathbb{R}^{n+n+n \times l+n \times d}\right)$, (A.1) also has a unique solution in $M^{2}\left(0, T ; \mathbb{R}^{n+n+n \times l+n \times d}\right)$.

Proof. Since for any $x \in \mathbb{R}^{n},\left(F_{0}, f_{0}, G_{0}, g_{0}\right) \in M^{2}\left(0, T ; \mathbb{R}^{n+n+n \times l+n \times d}\right), \varphi \in L^{2}\left(\Omega, \mathcal{F}_{T} ; \mathbb{R}^{n}\right)$, there exists a unique solution to (A.1) for $\alpha=\alpha_{0}$, thus for each $\bar{\zeta}=(\bar{y}, \bar{Y}, \bar{z}, \bar{Z}) \in M^{2}\left(0, T ; \mathbb{R}^{n+n+n \times l+n \times d}\right)$, there exists a unique quadruple $\zeta=(y, Y, z, Z) \in M^{2}\left(0, T ; \mathbb{R}^{n+n+n \times l+n \times d}\right)$ satisfying the following equations

$$
\left\{\begin{aligned}
\mathrm{d} y(t)= & {\left[f^{\alpha_{0}}(t, \zeta(t))+\delta(f(t, \bar{\zeta}(t))+\bar{y}(t))+f_{0}(t)\right] \mathrm{d} t-z(t) \overleftarrow{\mathrm{d}} B(t) } \\
& +\left[g^{\alpha_{0}}(t, \zeta(t))+\delta(g(t, \bar{\zeta}(t))+\bar{z}(t))+g_{0}(t)\right] \overrightarrow{\mathrm{d}} W(t), \\
\mathrm{d} Y(t)= & {\left[F^{\alpha_{0}}(t, \zeta(t))+\delta(F(t, \bar{\zeta}(t))+\bar{y}(t))+F_{0}(t)\right] \mathrm{d} t+Z(t) \overrightarrow{\mathrm{d}} W(t) } \\
& +\left[G^{\alpha_{0}}(t, \zeta(t))+\delta(G(t, \bar{\zeta}(t))+\bar{z}(t))+G_{0}(t)\right] \overleftarrow{\mathrm{d}} B(t), \\
y(0)= & x, \quad Y(T)=\Phi^{\alpha_{0}}(y(T))+\delta(\Phi(\bar{y}(T))-\bar{y}(T))+\varphi,
\end{aligned}\right.
$$

where $\delta$ is a positive number independent of $\alpha_{0}$ and less than 1 . We will prove that the mapping defined by

$$
\zeta=I_{\alpha_{0}+\delta}(\bar{\zeta}): M^{2}\left(0, T ; \mathbb{R}^{n+n+n \times l+n \times d}\right) \rightarrow M^{2}\left(0, T ; \mathbb{R}^{n+n+n \times l+n \times d}\right)
$$

is contractive for a small enough $\delta$. Let $\bar{\zeta}^{\prime}=\left(\bar{y}^{\prime}, \bar{Y}^{\prime}, \bar{z}^{\prime}, \bar{Z}^{\prime}\right) \in M^{2}\left(0, T ; \mathbb{R}^{n+n+n \times l+n \times d}\right)$ and $\zeta^{\prime}=\left(y^{\prime}, Y^{\prime}, z^{\prime}, Z^{\prime}\right)=$ $I_{\alpha_{0}+\delta}\left(\bar{\zeta}^{\prime}\right)$, and

$$
\begin{aligned}
& \widehat{\bar{\zeta}}=\bar{\zeta}-\bar{\zeta}^{\prime}=(\widehat{\bar{y}}, \widehat{\bar{Y}}, \widehat{\bar{z}}, \widehat{\bar{Z}})=\left(\bar{y}-\bar{y}^{\prime}, \bar{Y}-\bar{Y}^{\prime}, \bar{z}-\bar{z}^{\prime}, \bar{Z}-\bar{Z}^{\prime}\right), \\
& \widehat{\zeta}=\zeta-\zeta^{\prime}=(\widehat{y}, \widehat{Y}, \widehat{z}, \widehat{Z})=\left(y-y^{\prime}, Y-Y^{\prime}, z-z^{\prime}, Z-Z^{\prime}\right) .
\end{aligned}
$$


Applying Itô's formula to $\langle\widehat{y}, \widehat{Y}\rangle$ on $[0, T]$ and by virtue of (H2) and (H3), we have, since $\mathbb{E} \widehat{y}_{0}=0$,

$$
\begin{aligned}
\mathbb{E}\left\langle\widehat{y}(T), \alpha_{0}\left(\Phi(y(T))-\Phi\left(y(T)^{\prime}\right)\right)+(\right. & \left.\left.1-\alpha_{0}\right) \widehat{y}(T)+\delta\left(\Phi(\bar{y}(T))-\Phi\left(\bar{y}(T)^{\prime}\right)-\widehat{\bar{y}}(T)\right)\right\rangle \\
& \leq \mathbb{E} \int_{0}^{T}\left[\left(\alpha_{0}-\mu \alpha_{0}-1\right)|\widehat{\zeta}(t)|^{2}+\frac{\delta(k+1)}{2}|\widehat{\zeta}(t)|^{2}+\frac{\delta(k+1)}{2}|\widehat{\bar{\zeta}}(t)|^{2}\right] \mathrm{d} t .
\end{aligned}
$$

Then by virtue of (H3), we can derive that

$$
\left[\theta-\frac{\delta(k+1)}{2}\right] \mathbb{E} \int_{0}^{T}|\widehat{\zeta}(t)|^{2} \mathrm{~d} t \leq \frac{\delta(k+1)}{2} \mathbb{E} \int_{0}^{T}|\widehat{\bar{\zeta}}(t)|^{2} \mathrm{~d} t+\delta(k+1) \mathbb{E}|\widehat{y}(T)||\widehat{\bar{y}}(T)|,
$$

where $\theta=\min (1, \mu)$. Applying Itô's formula to $|\widehat{y}|^{2}$ on $[0, T]$ and by virtue of $(\mathrm{H} 2)$, by a standard method of estimation, we can derive that there exists a constant $c \geq 1$ which depends only on $k$, such that

$$
\mathbb{E}|\widehat{y}(T)|^{2} \leq c \mathbb{E} \int_{0}^{T}|\widehat{\zeta}(t)|^{2} \mathrm{~d} t+\delta c \mathbb{E} \int_{0}^{T}|\widehat{\bar{\zeta}}(t)|^{2} \mathrm{~d} t
$$

We now choose $\delta_{0}=\frac{2 \theta}{(4 c+1)(c+1)(k+1)}$. Then for any $\delta \in\left[0, \delta_{0}\right]$,

$$
\mathbb{E} \int_{0}^{T}|\widehat{\zeta}(t)|^{2} \mathrm{~d} t \leq \frac{1}{4 c}\left(\mathbb{E} \int_{0}^{T}|\widehat{\bar{\zeta}}(t)|^{2} \mathrm{~d} t+\mathbb{E}|\widehat{\bar{y}}(T)|^{2}\right) .
$$

Since $\delta_{0} \leq \frac{1}{4 c}$, we have for any $\delta \in\left[0, \delta_{0}\right]$,

$$
\mathbb{E}|\widehat{y}(T)|^{2} \leq \frac{1}{2}\left(\mathbb{E} \int_{0}^{T}|\widehat{\bar{\zeta}}(t)|^{2} \mathrm{~d} t+\mathbb{E}|\widehat{\bar{y}}(T)|^{2}\right) .
$$

It follows that, for each fixed $\delta \in\left[0, \delta_{0}\right]$, the mapping $I_{\alpha_{0}+\delta}$ is contractive in the following sense

$$
\mathbb{E} \int_{0}^{T}|\widehat{\zeta}(t)|^{2} \mathrm{~d} t+\mathbb{E}|\widehat{y}(T)|^{2} \leq \frac{3}{4}\left(\mathbb{E} \int_{0}^{T}|\widehat{\bar{\zeta}}(t)|^{2} \mathrm{~d} t+\mathbb{E}|\widehat{\bar{y}}(T)|^{2}\right) .
$$

Thus this mapping has a unique fixed point $\zeta=(y, Y, z, Z)$ in $M^{2}\left(0, T ; \mathbb{R}^{n+n+n \times l+n \times d}\right)$, which is the solution of (A.1) for $\alpha=\alpha_{0}+\delta$, as $\delta \in\left[0, \delta_{0}\right]$. The proof is complete.

Now we can give the proof of Proposition 2.1 - the existence and uniqueness theorem of (2.1).

Proof. (Uniqueness) Let $\zeta=(y, Y, z, Z)$ and $\zeta^{\prime}=\left(y^{\prime}, Y^{\prime}, z^{\prime}, Z^{\prime}\right)$ be two solutions of (2.1). We use the same notations as in Lemma A.2. Applying Itô's formula to $\langle\widehat{y}, \widehat{Y}\rangle$ on $[0, T]$, we have

$$
\mathbb{E}\langle\widehat{y}(T), \widehat{\Phi}(y(T))\rangle=\mathbb{E} \int_{0}^{T}\left\langle A(t, \zeta(t))-A\left(t, \zeta^{\prime}(t)\right), \widehat{\zeta}(t)\right\rangle \mathrm{d} t .
$$

By virtue of (H3), it follows that

$$
\mu \mathbb{E} \int_{0}^{T}\left\langle A(t, \zeta(t))-A\left(t, \zeta^{\prime}(t)\right), \widehat{\zeta}(t)\right\rangle \mathrm{d} t \leq 0 .
$$

Thus $\zeta \equiv \zeta^{\prime}$. The uniqueness is proven. 
(Existence). By Lemma A.1, for any $x \in \mathbb{R}^{n},\left(F_{0}, f_{0}, G_{0}, g_{0}\right) \in M^{2}\left(0, T ; \mathbb{R}^{n+n+n \times l+n \times d}\right), \varphi \in$ $L^{2}\left(\Omega, \mathcal{F}_{T} ; \mathbb{R}^{n}\right),\left(\right.$ A.1) has a unique solution in $M^{2}\left(0, T ; \mathbb{R}^{n+n+n \times l+n \times d}\right)$ as $\alpha=0$.

It follows from Lemma A.2 that there exists a positive constant $\delta_{0}=\delta_{0}(k, \mu)$ such that for any $\delta \in\left[0, \delta_{0}\right]$ and $x \in \mathbb{R}^{n}, \varphi \in L^{2}\left(\Omega, \mathcal{F}_{T} ; \mathbb{R}^{n}\right),\left(F_{0}, f_{0}, G_{0}, g_{0}\right) \in M^{2}\left(0, T ; \mathbb{R}^{n+n+n \times l+n \times d}\right)$, (A.1) has a unique solution for $\alpha=\delta$. Since $\delta_{0}$ depends only on $(k, \mu)$, we can repeat this process for $N$ times with $1 \leq N \delta_{0}<1+\delta_{0}$. In particular, for $\alpha=1$ with $\left(F_{0}, f_{0}, G_{0}, g_{0}\right) \equiv 0$ and $\varphi \equiv 0$, (A.1) has a unique solution in $M^{2}\left(0, T ; \mathbb{R}^{n+n+n \times l+n \times d}\right)$. The proof is complete.

Acknowledgements. The authors are grateful to the anonymous referees for their helpful comments and suggestions.

\section{REFERENCES}

[1] F. Baghery and B. Øksendal, A maximum principle for stochastic control with partial information. Stoch. Anal. Appl. 25 (2007) 705-717.

[2] S. Bahlali and B. Gherbal, Optimality conditions of controlled backward doubly stochastic differential equations. Random Oper. Stoch. Equ. 18 (2010) 247-265.

[3] A. Bally and A. Matoussi, Weak solutions of stochastic PDEs and backward doubly stochastic differential equations. J. Theoret. Probab. 14 (2001) 125-164.

[4] J.S. Baras, R.J. Elliott and M. Kohlmann, The partially-observed stochastic minimum principle. SIAM J. Control Optim. 27 (1989) 1279-1292.

[5] A. Bensoussan, Maximum principle and dynamic programming approaches of the optimal control of partially observed diffusions. Stochastics 9 (1983) 169-222.

[6] J.M. Bismut, An introductory approach to duality in optimal stochastic control. SIAM J. Control Optim. 20 (1978) 62-78.

[7] Y. Han, S. Peng and Z. Wu, Maximum principle for backward doubly stochastic control systems with applications. SIAM J. Control Optim. 48 (2010) 4224-4241.

[8] U.G. Haussmann, The maximum principle for optimal control of diffusions with partial information. SIAM J. Control Optim. 25 (1987) 341-361.

[9] L. Hu and Y. Ren, Stochastic PDIEs with nonlinear Neumann boundary conditions and generalized backward doubly stochastic differential equations driven by Lévy processes. J. Comput. Appl. Math. 229 (2009) 230-239.

[10] J. Huang, G. Wang and J. Xiong, A maximum principle for partial information backward stochastic control problems with applications. SIAM J. Control Optim. 48 (2009) 2106-2117.

[11] H.J. Kushner, Necessary conditions for continuous parameter stochastic optimization problems. SIAM J. Control Optim. 10 (1972) 550-565.

[12] X. Li and S. Tang, General necessary conditions for partially-observed optimal stochastic controls. J. Appl. Probab. 32 (1995) 1118-1137.

[13] Q. Meng, A maximum principle for optimal control problem of fully coupled forward-backward stochastic systems with partial information. Sci. China Ser. A $\mathbf{5 2}$ (2009) 1579-1588.

[14] Q. Meng and M. Tang, Necessary and sufficient conditions for optimal control of stochastic systems associated with Lévy processes. Sci. China Ser. F 52 (2009) 1982-1992.

[15] E. Pardoux and S. Peng, Backward doubly stochastic differential equations and systems of quasilinear parabolic SPDE's. Probab. Theory Related Fields 98 (1994) 209-227.

[16] S. Peng, A general stochastic maximum principle for optimal control problems. SIAM J. Control Optim. 28 (1990) 966-979.

[17] S. Peng and Y. Shi, A type of time-symmetric forward-backward stochastic differential equations. C. R. Acad. Sci. Paris, Ser. I 336 (2003) 773-778.

[18] L.S. Pontryagin, V.G. Boltyanskti, R.V. Gamkrelidze and E.F. Mischenko, The Mathematical Theory of Optimal Control Processe. Interscience, John Wiley, New York (1962).

[19] Y. Ren, A. Lin and L. Hu, Stochastic PDIEs and backward doubly stochastic differential equations driven by Lévy processes. J. Comput. Appl. Math. 223 (2009) 901-907.

[20] Y. Shi, Y. Gu and K. Liu, Comparison theorems of backward doubly stochastic differential equations and applications. Stoch. Anal. Appl. 23 (2005) 97-110.

[21] J. Shi and Z. Wu, The maximum principle for fully coupled forward backward stochastic control system. Acta Automat. Sinica 32 (2006) 161-169.

[22] J. Shi and Z. Wu, Maximum principle for partially-observed optimal control of fully-coupled forward-backward stochastic systems. J. Optim. Theory Appl. 145 (2010) 543-578.

[23] S. Tang, The maximum principle for partially observed optimal control of stochastic differential equations. SIAM J. Control Optim. 36 (1998) 1596-1617.

[24] G. Wang and $\mathrm{Z}$. Wu, The maximum principle for stochastic recursive optimal control problems under partial information. IEEE Trans. Autom. Control 54 (2009) 1230-1242. 
[25] Z. Wu, Maximum principle for optimal control problem of fully coupled forward-backward stochastic systems. Syst. Sci. Math. Sci. 11 (1998) 249-259.

[26] Z. Wu, A maximum principle for partially observed optimal control of forward-backward stochastic control systems. Sci. China Ser. F 53 (2010) 2205-2214.

[27] W. Xu, Stochastic maximum principle for optimal control problem of forward and backward system. J. Aust. Math. Soc. 37 (1995) 172-185.

[28] L. Zhang and Y. Shi, Maximum principle for forward-backward doubly stochastic control systems and applications. ESAIM: COCV 17 (2011) 1174-1197.

[29] L. Zhang and Y. Shi, Optimal Control of Stochastic Partial Differential Equations (2010) arXiv:1009.6061v2.

[30] Q. Zhang and H. Zhao, Pathwise stationary solutions of stochastic partial differential equations and backward doubly stochastic differential equations on infinite horizon. J. Funct. Anal. 252 (2007) 171-219.

[31] Q. Zhang and H. Zhao, Stationary solutions of SPDEs and infinite horizon BDSDEs under non-Lipschitz coefficients. J. Differ. Equations 248 (2010) 953-991.

[32] Q. Zhu, T. Wang and Y. Shi, Maximum principle for partially observed optimal control of backward doubly stochastic systems, in Proc. of the 30th Chinese Control Conference (2011) 1383-1388. 